\title{
An Analysis of Discrete Stage-Structured Prey and Prey-Predator Population Models
}

\author{
Arild Wikan \\ School of Business and Economics, The Arctic University of Norway, Campus Harstad, Harstad, Norway \\ Correspondence should be addressed to Arild Wikan; arild.wikan@uit.no
}

Received 23 February 2017; Accepted 27 March 2017; Published 16 April 2017

Academic Editor: Hammad Khalil

Copyright (C) 2017 Arild Wikan. This is an open access article distributed under the Creative Commons Attribution License, which permits unrestricted use, distribution, and reproduction in any medium, provided the original work is properly cited.

\begin{abstract}
Discrete stage-structured prey and prey-predator models are considered. Regarding the former, we prove that the models at hand are permanent (i.e., the population will neither go extinct nor exhibit explosive oscillations) and, moreover, that the transfer from stability to nonstationary behaviour always goes through a supercritical Neimark-Sacker bifurcation. The prey model covers species that possess a wide range of different life histories. Predation pressure may both stabilize and destabilize the prey dynamics but the strength of impact is closely related to life history. Indeed, if the prey possesses a precocious semelparous life history and exhibits chaotic oscillations, it is shown that increased predation may stabilize the dynamics and also, in case of large predation pressure, transfer the population to another chaotic regime.
\end{abstract}

\section{Introduction}

As it is well known, discrete nonlinear age- and stagestructured population models serve as excellent tools in order to study the dynamical outcomes of various ecological populations. Such models contain species which may possess different life histories ranging from biennials to species that may live for many years. Regarding the age-structured case, examples of studies on concrete species as well as pure theoretical approaches may be obtained in [1-7]. In the stagestructured case, we refer the reader to [8-13]. A comparison of dynamic outcomes from age- and stage-structured models may be obtained in [14], and the analysis of the models that also incorporate spatial structure may be found in $[15,16]$.

The models referred to above may also be extended to include prey-predator interactions (see [17-22]). Some of these models are two-dimensional in the sense that neither the prey nor the predator has an internal structure, while others may, for example, be four-dimensional. Considering the latter, Wikan [21] provides an analysis of several examples where both the prey and the predator populations are divided into two age classes.

The purpose of this paper is to bring out the analysis of a case where the predator preys upon newborns only.
Therefore, we have developed a three-dimensional model where we split the prey population into two separate parts by use of the same strategy as in [10], while the interaction between newborn prey and the predator is modelled in the same way as in [18]. The focus is on the stability and nonlinear behaviour. In particular, we address the question how the impact from the predator acts on prey populations that possess different life histories. The results from analysis of the prey part of the model are also provided and compared with findings obtained from other models.

The structure of the paper is as follows: In Section 2, we present and analyse the various models with respect to stability and nonstationary behaviour. Section 3 provides examples of prey models as well as prey-predator interactions while in Section 4 we unify and discuss the results.

\section{Model(s), Fixed Points, and Stability}

Let $x_{1, t}$ and $x_{2, t}$ be the immature and mature part of the prey population $x_{t}=x_{1, t}+x_{2, t}$ at time $t$, respectively, and let $y_{t}$ be the predator population. $\mu_{1}$ and $\mu_{2}$, such that $0<\mu_{1}$ and $\mu_{2}<1$, are the fractions of the immature population $x_{1}$ and the mature (adult) population $x_{2}$ that survive from time $t$ to time $t+1$. $p$, with $0<p<1$, is the fraction 
of the immature population that survives to become mature one time unit (year) later and $f=F \exp \left(-x_{2}\right), F>0$, is the density dependent fecundity. Depending on the species under consideration, $\exp \left(-x_{2}\right)$ may account for crowding effects, effects linked to shortage of food, and for some species it may also incorporate cannibalistic behaviour. Further, it is assumed that predation will take place only on the young of the part year, $\exp \left(-x_{2}\right) x_{2}$, of the prey population. This is accounted for by the term $\exp (-a y)$ where $a \geq 0$ measures the skill of predation. The constant $c, c>0$, may be interpreted as a conversion of prey into predator, or clutch parameter, the following year (see [18]). The relation between $x_{1}, x_{2}$, and $y$ at two consecutive time steps may then be given as a map:

$$
\begin{aligned}
& \left(x_{1}, x_{2}, y\right) \longrightarrow\left(\mu_{1}(1-p) x_{1}\right. \\
& \quad+F \exp \left(-x_{2}\right) \exp (-a y) x_{2}, \mu_{1} p x_{1} \\
& \left.\quad+\mu_{2} x_{2} c F \exp \left(-x_{2}\right) x_{2}(1-\exp (-a y))\right) .
\end{aligned}
$$

When $a \rightarrow 0$, (1) degenerates to the prey map:

$$
\begin{aligned}
& \left(x_{1}, x_{2}\right) \\
& \quad \longrightarrow\left(\mu_{1}(1-p) x_{1}+F \exp \left(-x_{2}\right) x_{2}, \mu_{1} p x_{1}+\mu_{2} x_{2}\right) .
\end{aligned}
$$

Note that (2) has a striking similarity to the general stagestructured model proposed and analysed in [10]. The difference is found in the density dependent term.

By adjusting the size of the parameters, it is easy to see that (2) covers species that possess different life histories. Indeed, following [10], if $\mu_{2} \rightarrow 0$, the population is semelparous (i.e., reproducing only once). If $0<\mu_{2}<1$, the population is iteroparous (repeated reproduction). The subclass $\mu_{2} \rightarrow 0$, $p \rightarrow 1$ is often referred to as precocious semelparity which covers species with rapid development followed by only one reproduction, for example, biennials and annual plants (see [11]). Delayed semelparity occurs when $\mu_{2} \rightarrow 0$ and $0<p<$ 1 . Typical examples are periodical cicadas $[11,23]$, and several salmon species that live for many years before they become mature and reproduce only once. We may also divide the iteroparous case into two subclasses. The subclass $0<\mu_{2}<1$, $p \rightarrow 1$, is classified as precocious iteroparity and covers several small mammals species, among them small rodent species that start to reproduce at young age and may survive to reproduce for several years. The fourth subclass, delayed iteroparity, is characterized by $0<\mu_{2}<1,0<p<1$, which covers species which may live long before maturity and then survive to reproduce for many years. In this subclass, we find large mammals. Consequently, (2) may be used in order to capture the dynamics of a wide range of (prey) populations, and the role and impact of predation on newborns and young individuals are then analysed by (1).

We start by revealing some properties of (2). There are two fixed points, the trivial one $\left(\widetilde{x}_{1}, \tilde{x}_{2}\right)=(0,0)$ and the nontrivial one:

$$
\left(x_{1}^{*}, x_{2}^{*}\right)=\left(\frac{1-\mu_{2}}{\mu_{1} p} K, K\right) \text {, }
$$

where

$$
K=\ln \left(\frac{\mu_{1} p F}{\left(1-\mu_{2}\right)\left(1-\mu_{1}(1-p)\right)}\right)=\ln R_{0},
$$

and in order for (3) to be a feasible fixed point (equilibrium), we assume $R_{0}>1$. Moreover, by use of stability analysis, it is straightforward to show that $\left(\tilde{x}_{1}, \tilde{x}_{2}\right)$ is stable provided $R_{0} \leq 1$. Therefore, the restriction $R_{0}>1$ ensures both that the origin is a repeller and that (3) is feasible.

Model (2) is said to be permanent if there exist $\delta>0$ and $D>0$ such that

$$
\delta<\liminf _{t \rightarrow \infty} x_{t} \leq \lim _{t \rightarrow \infty} \sup x_{t}<D
$$

(cf. [24]).

Theorem 1. Model (2) is permanent provided $R_{0}>1$.

Proof. See Appendix A.

Considering the stability properties of the nontrivial fixed point (3), we find that the eigenvalue equation of the linearization of (2) may be expressed as

$$
\lambda^{2}+a_{1} \lambda+a_{2}=0
$$

where

$$
\begin{aligned}
& a_{1}=-\left(\mu_{1}(1-p)+\mu_{2}\right) \\
& a_{2}=\mu_{1}(1-p) \mu_{2}-(1-K)\left(1-\mu_{2}\right)\left(1-\mu_{1}(1-p)\right),
\end{aligned}
$$

and according to the Jury criteria (cf. [25]), (3) is stable provided the inequalities $1+a_{1}+a_{2}>0,1-a_{2}+a_{3}>0$, and $1-\left|a_{2}\right|>0$ hold; that is,

$$
\begin{aligned}
& \left(1-\mu_{2}\right)\left(1-\mu_{1}(1-p)\right) K>0, \\
& 2\left(\mu_{1}(1-p)+\mu_{2}\right)+K\left(1-\mu_{2}\right)\left(1-\mu_{1}(1-p)\right)>0, \\
& K=\ln R_{0}<\frac{2-\left(\mu_{1}(1-p)+\mu_{2}\right)}{\left(1-\mu_{2}\right)\left(1-\mu_{1}(1-p)\right)} .
\end{aligned}
$$

Obviously, the left-hand sides, LHS, of (8a) and (8b) are positive. Therefore, there will be no transfer from stability to instability as an eigenvalue crosses the boundary of the unit circle through $1(8 \mathrm{a})$ or $-1(8 \mathrm{~b})$. Regarding (8c), it is clearly valid for small values of $K$ but when $K$ is increased (as a result of increasing $F$ ), LHS of ( $8 c$ ) eventually equals the right-hand side, RHS, and $\left(x_{1}^{*}, x_{2}^{*}\right)$ will lose its hyperbolicity through a Neimark-Sacker (Hopf) bifurcation as a pair of complex valued eigenvalues cross the unit circle. Such a bifurcation may be of supercritical or subcritical nature. In the former case, when the fixed point loses its stability, an attracting invariant curve about the unstable fixed point is established and the dynamics are restricted to that curve. In the latter case (subcritical), there is no such attracting curve. Now, considering (2), we have the following result.

Theorem 2. Consider (2) together with the fixed point $\left(x_{1}^{*}, x_{2}^{*}\right)$ given by (3). Then, for the fixed values of $\mu_{1}, \mu_{2}$ and $p, 0<$ 
TABLE 1

\begin{tabular}{llllllllllll}
\hline$a$ & 0.4 & 0.5 & 0.56 & 0.6 & 0.7 & 0.76 & 0.80 & 1 & 1.5 & 2 \\
\hline$y^{*}$ & $\approx 0$ & $\approx 0$ & 0.512 & 1.286 & 1.655 & 1.682 & 1.674 & 1.530 & 1.148 & 0.901 & 0.625 \\
$a y^{*}$ & $\approx 0$ & $\approx 0$ & 0.287 & 0.772 & 1.158 & 1.278 & 1.339 & 1.530 & 1.723 & 1.802 & 1.875 \\
$x_{2}^{*}$ & $\approx 2$ & $\approx 2$ & 1.713 & 1.228 & 0.842 & 0.722 & 0.661 & 0.470 & 0.277 & 0.198 & 0.125 \\
$x_{1}^{*}$ & $\approx 2$ & $\approx 2$ & 1.713 & 1.228 & 0.842 & 0.722 & 0.661 & 0.470 & 0.277 & 0.198 & 0.125 \\
\hline
\end{tabular}

TABLE 2

\begin{tabular}{lcccccccccc}
\hline$a$ & 1.00 & 1.05 & 1.10 & 1.20 & 1.30 & 1.40 & 1.50 & 1.60 & 2.00 & 3.00 \\
\hline$y^{*}$ & $\approx 0$ & 1.384 & 1.705 & 1.825 & 1.804 & 1.749 & 1.682 & 1.614 & 1.364 & 0.961 \\
$a y^{*}$ & $\approx 0$ & 1.453 & 1.875 & 2.190 & 2.345 & 2.448 & 2.523 & 2.582 & 2.729 & 2.882 \\
$x_{2}^{*}$ & 3.11 & 1.657 & 1.235 & 0.920 & 0.765 & 0.662 & 0.587 & 0.528 & 0.381 & 0.228 \\
$x_{1}^{*}$ & 1.729 & 0.921 & 0.687 & 0.511 & 0.425 & 0.368 & 0.326 & 0.294 & 0.212 & 0.127 \\
\hline
\end{tabular}

$\mu_{1}, \mu_{2}, p<1$, (3) will undergo a supercritical Neimark-Sacker bifurcation at the threshold

$$
K=\ln R_{0}=\frac{2-\left(\mu_{1}(1-p)+\mu_{2}\right)}{\left(1-\mu_{2}\right)\left(1-\mu_{1}(1-p)\right)}
$$

or equivalently when

$$
\begin{gathered}
F=F_{c}=\frac{\left(1-\mu_{2}\right)\left(1-\mu_{1}(1-p)\right)}{\mu_{1} p} \\
\cdot \exp \left(\frac{2-\left(\mu_{1}(1-p)+\mu_{2}\right)}{\left(1-\mu_{2}\right)\left(1-\mu_{1}(1-p)\right)}\right) .
\end{gathered}
$$

Proof. See Appendix B.

Next, let us focus on the "full" prey-predator map (1). There is one obvious fixed point, namely, the trivial one $\left(\tilde{x}_{1}, \tilde{x}_{2}, \tilde{y}\right)=(0,0,0)$. The other fixed point is in the form of

$$
\left(x_{1}^{*}, x_{2}^{*}, y^{*}\right)=\left(\frac{1-\mu_{2}}{\mu_{1} p}\left(K-a y^{*}\right), K-a y^{*}, y^{*}\right),
$$

where $y^{*}$ must be found by means of numerical methods from the equation

$$
y-c \frac{\left(1-\mu_{2}\right)\left(1-\mu_{1}(1-p)\right)}{\mu_{1} p}(K-a y)\left(e^{a y}-1\right)=0 .
$$

Clearly, if $a=0$, (11) implies that $y=0$ so one possibility is that $(10)$ is in the form of $\left(x_{1}^{*}, x_{2}^{*}, 0\right)$. Depending on the value of $K$, the same scenario persists also in the case of $a>$ 0 . Consequently, as also found in the prey-predator model analysed in [21], the interaction or skill parameter a must exceed a critical threshold $a_{c}$ in order to establish a fixed point $\left(x_{1}^{*}, x_{2}^{*}, y^{*}\right)$ where both species coexist. Moreover, note that $a<a_{c}$ implies $a y^{*}=0$. If $a>a_{c}$, an increase of a makes $a y^{*}$ larger which according to (10) leads to a reduction of $x_{2}^{*}$ and $x_{1}^{*}$. A final observation from (10) and (11) is that a decrease of clutch parameter $c$ leads to a smaller predator equilibrium $y^{*}$.
This makes sense; the smaller the $c$, the smaller the benefit of eating. A couple of numerical examples are presented in Tables 1 and 2 . In Table 1, we have used the parameter values $\mu_{1}=1, \mu_{2}=0.1, p=0.9, c=1$, and $K=2(F=6.65)$ which means that the prey possesses precocious semelparous life history and that the prey in the absence of the predator has a stable fixed point $\left(x_{1}^{*}, x_{2}^{*}\right)$. In Table 2 , the prey may be classified as a precocious iteroparous population. Parameter values are $\mu_{1}=1, \mu_{2}=0.5, p=0.9, c=0.5, K=$ $3.11(F=11.22)$ which means that $\left(x_{1}^{*}, x_{2}^{*}\right)$ is located at instability threshold.

Now, let us turn to stability. The eigenvalue equation of the linearization of (1) may be expressed as

$$
\lambda^{3}+a_{1} \lambda^{2}+a_{2} \lambda+a_{3}=0
$$

where the coefficients are

$$
\begin{aligned}
a_{1}= & -\left\{\mu_{1}(1-p)+\mu_{2}+a c F x_{2}^{*} e^{-K}\right\} \\
a_{2}= & \mu_{1}(1-p) \mu_{2}+\mu_{1}(1-p) a c F x_{2}^{*} e^{-K} \\
& +a c \mu_{2} F x_{2}^{*} e^{-K}-\mu_{1} p F\left(1-x_{2}^{*}\right) e^{-K} \\
a_{3}= & \mu_{1} p a c F^{2}\left(1-x_{2}^{*}\right) x_{2}^{*} e^{-x_{2}^{*}} e^{-K} \\
& -\mu_{1}(1-p) \mu_{2} a c F x_{2}^{*} e^{-K} .
\end{aligned}
$$

As long as all eigenvalues of (12) are located within the unit circle, (10) is stable and this is ensured if the Jury criteria

$$
\begin{aligned}
1+a_{1}+a_{2}+a_{3} & >0 \\
1-a_{1}+a_{2}-a_{3} & >0 \\
1-\left|a_{2}\right| & >0 \\
\left|1-a_{3}^{2}\right|-\left|a_{2}-a_{3} a_{1}\right| & >0
\end{aligned}
$$

hold. 
Now, by use of the relation $x_{2}^{*}+a y^{*}=K$ and map (1), we find that criteria (14a)-(14d) may be cast in the forms

$$
\begin{aligned}
& \left(1-\mu_{2}\right)\left(1-\mu_{1}(1-p)\right)\left[K-\left(K-a y^{*}\right) \frac{a y^{*}}{1-e^{-a y^{*}}}\right] \\
& >0 \\
& \left(1+\mu_{2}\right)\left(1+\mu_{1}(1-p)\right)\left(1+\frac{a y^{*}}{1-e^{-a y^{*}}} e^{-a y^{*}}\right)-(1 \\
& \left.-\mu_{2}\right)\left(1-\mu_{1}(1-p)\right)\left(1-\left(K-a y^{*}\right)\right)(1 \\
& \left.+\frac{a y^{*}}{1-e^{-a y^{*}}}\right)>0 \\
& 1-\frac{a y^{*}}{1-e^{-a y^{*}}}\left[\left(1-\mu_{2}\right)\left(1-\mu_{1}(1-p)\right)(1\right. \\
& \left.\left.-\left(K-a y^{*}\right)\right)-\mu_{1}(1-p) \mu_{2} e^{-a y^{*}}\right]>0 \\
& \left(1-\mu_{1}(1-p) \mu_{2}\right)\left\{1-\frac{a y^{*}}{1-e^{-a y^{*}}} e^{-a y^{*}}\left[\mu_{1}(1-p)\right.\right. \\
& \left.\left.+\mu_{2}+\mu_{1}(1-p) \mu_{2} \frac{a y^{*}}{1-e^{-a y^{*}}} e^{-a y^{*}}\right]\right\}+\left(1-\mu_{2}\right) \\
& \cdot\left(1-\mu_{1}(1-p)\right)\left(1-\left(K-a y^{*}\right)\right) \times\{1 \\
& -\frac{a y^{*}}{1-e^{-a y^{*}}}\left(\mu_{1}(1-p)+\mu_{2}\right)+\left(\frac{a y^{*}}{1-e^{-a y^{*}}}\right)^{2} \\
& \frac{e^{-a y^{*}}}{K-a y^{*}}\left[\mu_{1}(1-p) \mu_{2}\right. \\
& \left.-\left(K-a y^{*}\right)\left(1-\mu_{1}(1-p) \mu_{2}\right)\right]-\left(\frac{a y^{*}}{1-e^{-a y^{*}}}\right)^{2} \\
& \left.\cdot\left(1-\mu_{2}\right)\left(1-\mu_{1}(1-p)\right) \frac{1-\left(K-a y^{*}\right)}{K-a y^{*}}\right\}>0
\end{aligned}
$$

and our first observation is that the clutch parameter $c$ drops out of the criteria. Thus, different values of $c$ correspond to different values of $x_{1}^{*}, x_{2}^{*}$, and $y^{*}$ but it does not affect qualitative changes of dynamics.

Considering the criteria, assuming $a>a_{c}$, we find that LHS of (15a) is an increasing function of $a y^{*}$ provided

$$
a y^{*}+\frac{a y^{*}\left(1-e^{-a y^{*}}\right)}{1-\left(1+a y^{*}\right) e^{-a y^{*}}} \geq K .
$$

The minimal value is found to be

$$
\begin{aligned}
& \left(1-\mu_{2}\right)\left(1-\mu_{1}(1-p)\right) \\
& \cdot \frac{2 a y^{*}\left(1-e^{-a y^{*}}\right)-\left(a y^{*}\right)^{2}\left(1+e^{-a y^{*}}\right)}{1-\left(1+a y^{*}\right) e^{-a y^{*}}}
\end{aligned}
$$

and the sign as well as the value of (17) depends on $K$.
Turning to (15b), we find that LHS is a decreasing function since $g(z)=z e^{-z}\left(1-e^{-z}\right)^{-1}$ is decreasing and $(1-(K-z))$ as well as $z\left(1-e^{-z}\right)^{-1}$ is increasing. Further, observe that in case of $a y^{*} \rightarrow 0$ the LHS approaches the positive expression

$$
\begin{aligned}
& 2\left[\left(1+\mu_{2}\right)\left(1+\mu_{1}(1+p)\right)\right. \\
& \left.\quad-\left(1-\mu_{2}\right)\left(1-\mu_{1}(1-p)\right)(1-K)\right] .
\end{aligned}
$$

On the other hand, if $a y^{*} \rightarrow K$, the LHS may be expressed as

$$
\begin{aligned}
& \left(1+\mu_{2}\right)\left(1+\mu_{1}(1-p)\right) \\
& \quad-\left(1-\mu_{2}\right)\left(1-\mu_{1}(1-p)\right) \frac{K}{e^{-K}}
\end{aligned}
$$

which is negative provided $K$ is large enough.

Scrutinizing (15c), the same picture emerges. LHS is a decreasing function and when $a y^{*}$ is small, LHS approaches the positive expression

$$
1-\left(1-\mu_{2}\right)\left(1-\mu_{1}(1-p)\right)(1-K)+\mu_{1}(1-p) \mu_{2} .
$$

When $a y^{*}$ becomes large, LHS degenerates to

$$
\begin{aligned}
1- & \frac{K}{1-e^{-K}}\left[\left(1-\mu_{2}\right)\left(1-\mu_{1}(1-p)\right)\right. \\
& \left.-\mu_{1}(1-p) \mu_{2} e^{-K}\right]
\end{aligned}
$$

which is negative. Now, assuming equality in (15c), we find

$$
\begin{aligned}
1+ & \frac{a y^{*}}{1-e^{-a y^{*}}} e^{-a y^{*}} \mu_{1}(1-p) \mu_{2}=\frac{a y^{*}}{1-e^{-a y^{*}}}\left(1-\mu_{2}\right) \\
& \cdot\left(1-\mu_{1}(1-p)\right)\left(1-\left(K-a y^{*}\right)\right)
\end{aligned}
$$

and by substituting into (15b) we arrive at

$$
\begin{aligned}
& \mu_{1}(1-p)+\mu_{2}+\mu_{1}(1-p) \mu_{2} \\
& \quad+\frac{a y^{*}}{e^{a y^{*}}-1}\left[1+\mu_{2}-\mu_{1}(1-p)\left(1+2 \mu_{2}\right)\right] \\
& \quad-\left(1-\mu_{2}\right)\left(1-\mu_{1}(1-p)\right)\left(1-\left(K-a y^{*}\right)\right)>0
\end{aligned}
$$

which holds. Consequently, LHS of (15c) crosses the $a y^{*}$ axis before LHS of (15b) so we may rule out the possibility of a period doubling bifurcation at instability threshold.

Finally, turning to (15d), when $a y^{*} \rightarrow K$, the LHS becomes negative (notice the term $K-a y^{*}$ in the last fraction). In the case $a y^{*} \rightarrow 0$, the LHS may be written as

$$
\begin{aligned}
& \left(1-\mu_{1}(1-p) \mu_{2}\right)\left\{1-\left[\mu_{1}(1-p)+\mu_{2}\right.\right. \\
& \left.\left.\quad+\mu_{1}(1-p) \mu_{2}\right]\right\}+\left(1-\mu_{2}\right)\left(1-\mu_{1}(1-p)\right)(1 \\
& \quad-K) \times\left\{\frac { 1 } { K } \left[\mu_{1}(1-p) \mu_{2}\right.\right. \\
& \left.\quad-\left(1-\mu_{2}\right)\left(1-\mu_{1}(1-p)\right)(1-K)\right]-\mu_{1}(1-p) \\
& \left.\quad \cdot\left(1-\mu_{2}\right)-\mu_{2}\right\} .
\end{aligned}
$$




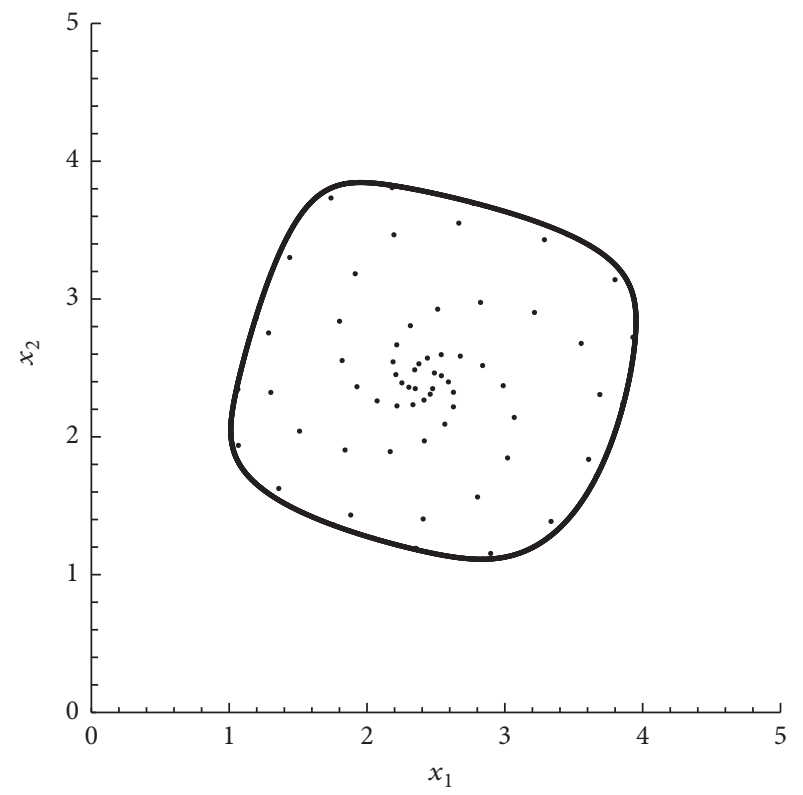

(a)

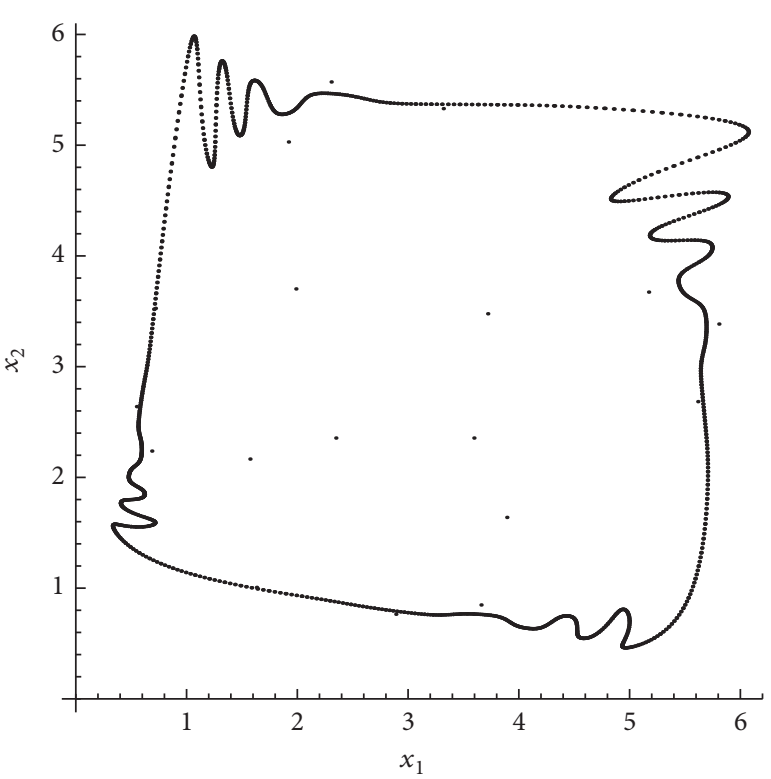

(b)

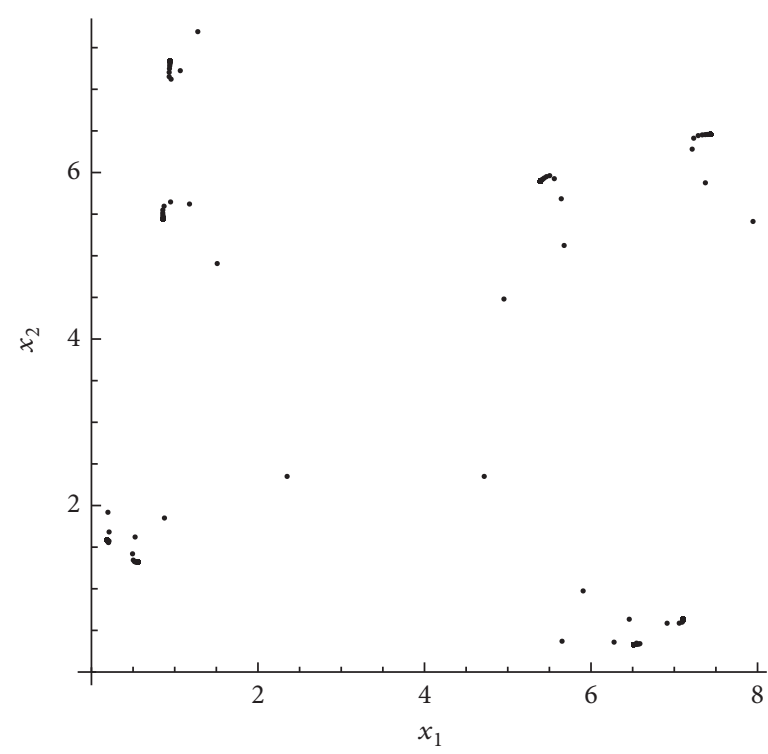

(c)

Figure 1: Dynamics generated by (2). Parameter values $\mu_{1}=1, p=0.9$, and $\mu_{2}=0.1$. (a) $F=10$, (b) $F=15$, and (c) $F=20$.

This expression is positive for small values of $K$ and approaches zero when $K$ becomes large.

Thus, to summarise, if $K$ is so small that the prey in the absence of the predator possesses a stable fixed point $\left(x_{1}^{*}, x_{2}^{*}\right)$, all LHS of (15a)-(15d) are positive. Whenever the population pressure ay is small, the long term dynamics is a stable fixed point $\left(x_{1}^{*}, x_{2}^{*}, y^{*}\right)$. As predation pressure ay increases, the magnitude of the dominating eigenvalue(s) becomes smaller until the graph of LHS of (15a) intersects the corresponding graph of (15d) or (15c). Then, the magnitude increases and the fixed point undergoes a Neimark-Sacker bifurcation when the LHS of (15d) or (15c) becomes zero. If $K$ is so large that the prey in the absence of the predator exhibits nonstationary dynamics, (17) is negative in case of $a y^{*}$ small; therefore, $\left(x_{1}^{*}, x_{2}^{*}, y^{*}\right)$ is unstable. However, when $a y^{*}$ increases, we observe the same qualitative picture as reported above.

\section{Examples}

First, we concentrate on dynamics generated by the "prey map" (2) and we start with a numerical example where $\mu_{1}=$ $1, p=0.9$, and $\mu_{2}=0.1$ (precocious semelparity). At threshold (9b), $F=8.31$ (or $K=2.22$ ) and the fixed point $\left(x_{1}^{*}, x_{2}^{*}\right)=(2.22,2.22)$ undergoes a Neimark-Sacker bifurcation. In Figure 1(a), we show the attracting invariant curve together with some initial transients in case of $F=10$. 
When $F$ is further increased $(F=15)$, the curve becomes kinked and not topologically equivalent to a circle anymore and in case of $F=20$ the curve has broken up into a diffuse cloud of points. Thus, through an enlargement of $F$ (or $K$ ), the dynamics change from stability to chaotic behaviour. The whole scenario is displayed in Figures 1(a)-1(c).

If we instead use $\mu_{1}=1, p=0.3$, and $\mu_{2}=0.1$ (delayed semelparity), $F=33.31$ at threshold and the corresponding fixed point is $\left(x_{1}^{*}, x_{2}^{*}\right)=(8.12,3.61)$. When $F>33.31$, we find invariant curves here too, and such a curve also persists when $F>100$. We have not detected chaotic dynamics as in the previous case. Hence, a natural conclusion to suggest is that populations that possess delayed semelparous life histories have better stability properties than populations with precocious semelparous life histories. This is in excellent agreement with results obtained by Neubert and Caswell [10] but not with the findings in [14] where an age-structured model is analysed.

Turning to the precocious iteroparous case, exemplified by $\mu_{1}=1, p=0.9$, and $\mu_{2}=0.5$, we find $F=11.22$ and $\left(x_{1}^{*}, x_{2}^{*}\right)=(1.72,3.11)$ at instability threshold. Beyond threshold, the behaviour is qualitatively similar to the precocious semelparous case.

Finally, considering delayed iteroparity through the example of $\mu_{1}=1, p=0.4$, and $\mu_{2}=0.5$, we find from (9b) that $F=45$ at threshold and the corresponding fixed point becomes $\left(x_{1}^{*}, x_{2}^{*}\right)=(5.63,4.51)$. As we increase $F$ beyond 45 , there are invariant curves with the same kind of shapes as in the former case. The main difference between the precocious and delayed case is the size of equilibrium population at threshold. Hence, by use of the same classification as in [10], we find it natural to support the conjecture that species that possess a delayed iteroparous life history have better stability properties than populations with precocious iteroparous life histories.

Next, consider the "full" prey-predator map (1). We start our analysis by use of the same parameter values as we did when Table 1 was established (i.e., we study a case where the prey possesses a precocious semelparous life history). As already shown, $K=2$ implies that $\left(x_{1}^{*}, x_{2}^{*}\right)$ is stable in the absence of predation. Then, we increase ay (starting at $a y=$ 0.28 , cf. Table 1) and calculate the LHS of (15a)-(15d), respectively, and the results of these calculations are presented in Figure 2. Clearly, $\left(x_{1}^{*}, x_{2}^{*}, y^{*}\right)$ is stable in the interval $0.28 \leq$ ay $\leq 1$.43. It fails to be stable when LHS of (15d) becomes zero and a (supercritical) Neimark-Sacker bifurcation takes place. Just beyond 1.43 the nonstationary dynamics are restricted to an invariant curve, but through further enlargement of ay the curve becomes kinked and eventually it breaks up. This is shown in Figures 3(a)-3(c). Thus, in case of small and moderate predation stability properties are improved. On the other hand, severe predation pressure may be classified as a strong destabilizing effect.

The results referred to above are further strengthened through our next example. In Figure 4, we present the calculations of LHS of (15a)-(15d) in case of $K=2.5$ (which in contrast to the previous example means that $\left(x_{1}^{*}, x_{2}^{*}\right)$ is unstable in case of no predation). As is shown, there is no stable fixed point in the interval $0.4 \leq a y \leq 0.71$

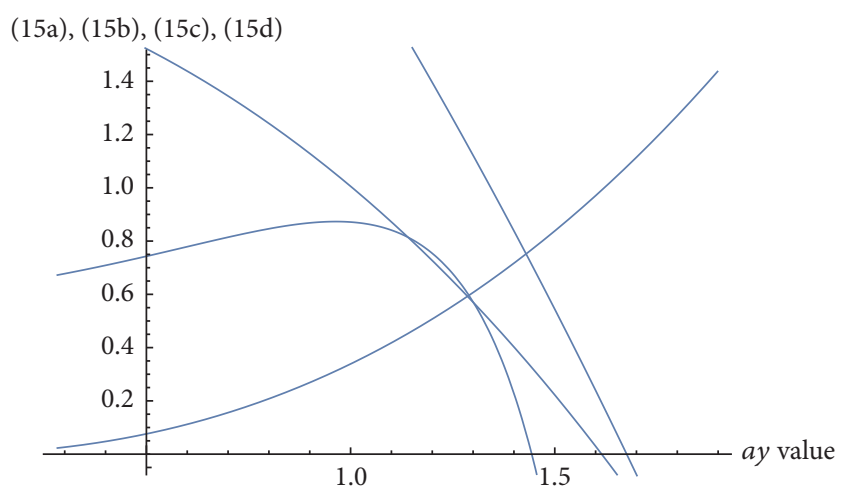

Figure 2: The graphs of LHS of (15a), (15b), (15c), and (15d). Parameter values: $\mu_{1}=c=1, p=0.9, \mu_{2}=0.1$, and $K=2$. LHS of (15a) is the increasing function. LHS of (15d), (15c), and (15b) cross the $a y$-axis at $1.44,1.61$, and 1.67 , respectively.

(cf. (16) and (17)). Otherwise, the graphs look similar to the graphs in Figure 2. In Figure 5, we display the dynamics. In Figure 5(a), we use $a=0.425$, and Figures 5(b) and 5(c) show the cases $a=0.75$ and $a=1.5$.

Both examples referred to above consider species that possess a precocious semelparous life history. We have also scrutinised species with other life histories and our findings are that there is a striking resemblance between the graphs of (15a)-(15d) in these cases and Figures 2 and 4. The main difference really is that ay must be increased to a much higher value in the delayed iteroparous case in order to establish an equilibrium with coexisting species.

\section{Discussion}

In the previous section, we have analysed a selected number of stage-structured prey and prey-predator models. The parameter space is huge which allows us to consider prey populations that possess a wide range of different life histories. We shall now unify and discuss our findings.

First, let us comment on the prey map (2). As is shown, independent of life history, (2) possesses a stable fixed point $\left(x_{1}^{*}, x_{2}^{*}\right)$ whenever $F$ is small enough and the transfer from stability to instability occurs when the fixed point undergoes a supercritical Neimark-Sacker bifurcation at thresholds (9a) and (9b). In Figure 6, we show the value of the total equilibrium population $x^{*}=x^{*}(p)$ at instability for different values of $\mu_{2}$. For a given value of $\mu_{2}$, the stable region is located below the corresponding curve. Hence, whenever $p>0.3$ (roughly), populations which exhibit iteroparous life histories have better stability properties than species that possess semelparous life histories. On the other hand, if $p<$ 0.3 , we arrive at the opposite conclusion. Moreover, since all functions $x^{*}(p)$ are decreasing, $\left(x^{*^{\prime}}(p)=-\mu_{1}\left(1-\mu_{1}(1-\right.\right.$ $p))^{-2}$ ), we may also conclude that the delayed cases appear to be more stable than the precocious ones.

It is tempting to compare the findings above with the results obtained from the model:

$$
\left(x_{1}, x_{2}\right) \longrightarrow\left(\mu_{1}(1-p) x_{1}+F e^{-x} x_{2}, \mu_{1} p x_{1}+\mu_{2} x_{2}\right)
$$




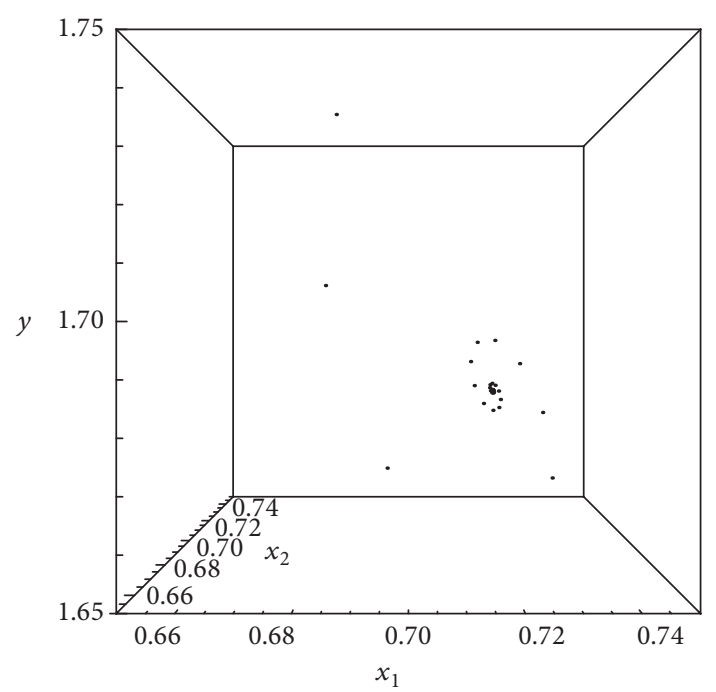

(a)

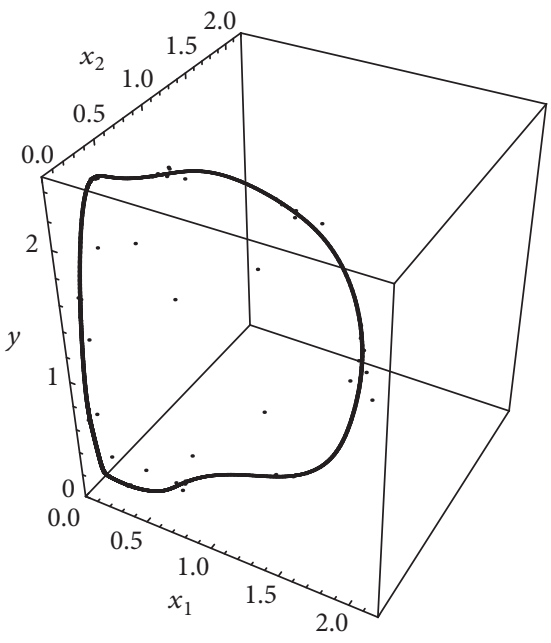

(b)

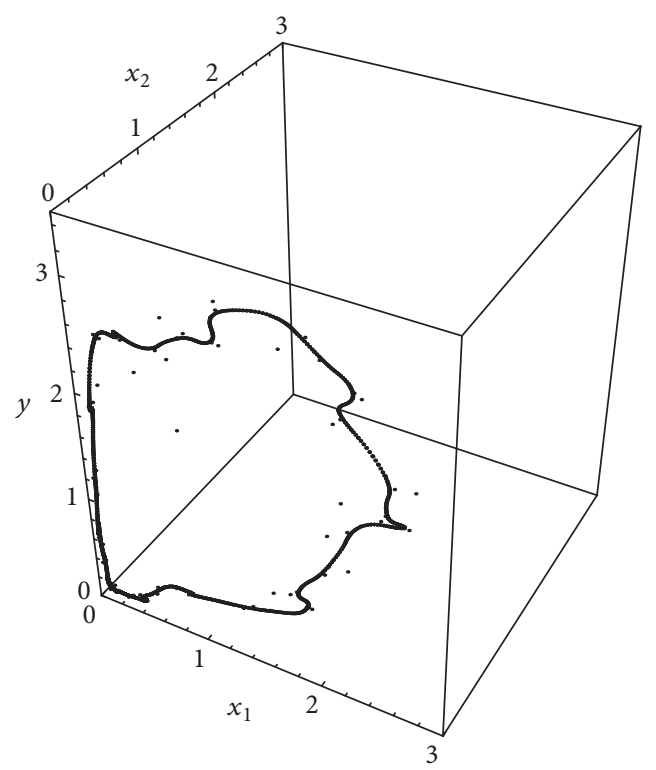

(c)

Figure 3: Dynamics generated by (1). Parameter values: $\mu_{1}=c=1.0, p=0.9, \mu_{2}=0.1$, and $K=2$. (a) $a=0.76$, (b) $a=1.6$, and (c) $a=2$.

(15a), (15b), (15c), (15d)

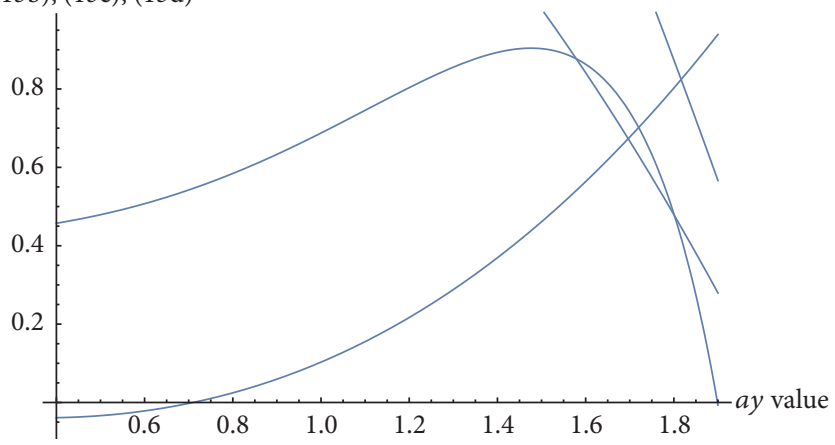

Figure 4: LHS of (15a)-(15d) in case of $K=2.5$. Other parameter values as in Figure 2. 


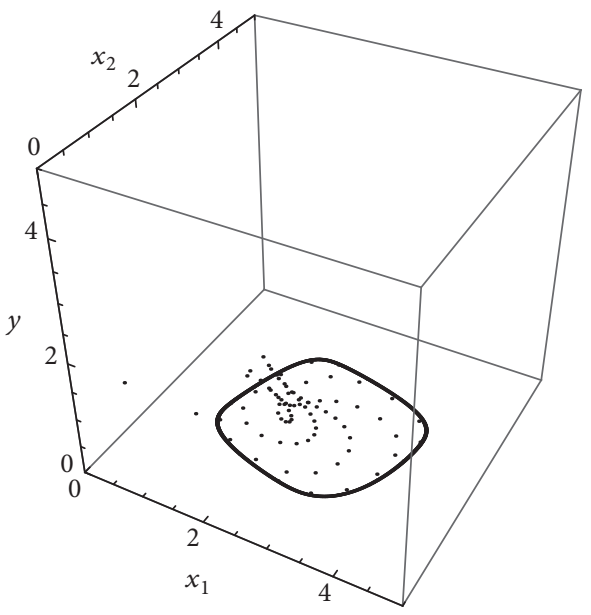

(a)

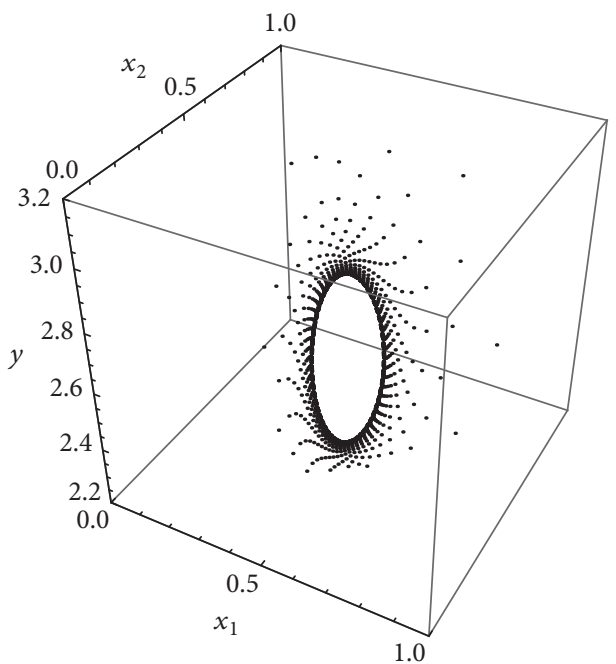

(b)

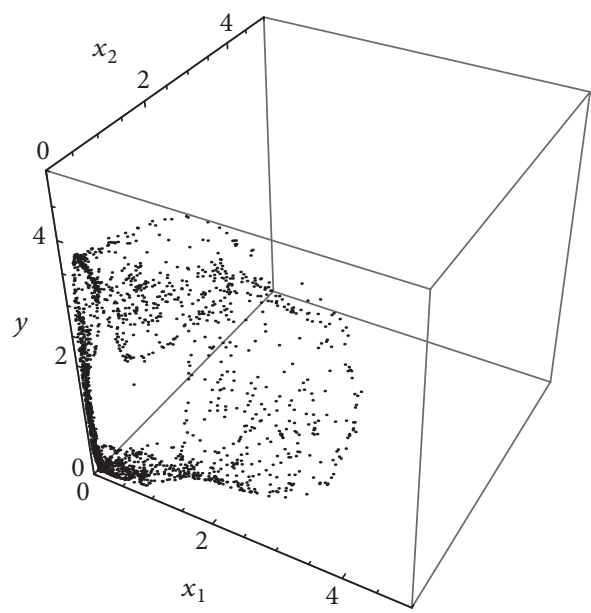

(c)

Figure 5: Dynamics generated by (1). Parameter values as in Figure 3 except $K=2.5$. (a) $a=0.425$, (b) $a=0.75$, and (c) $a=1.5$.

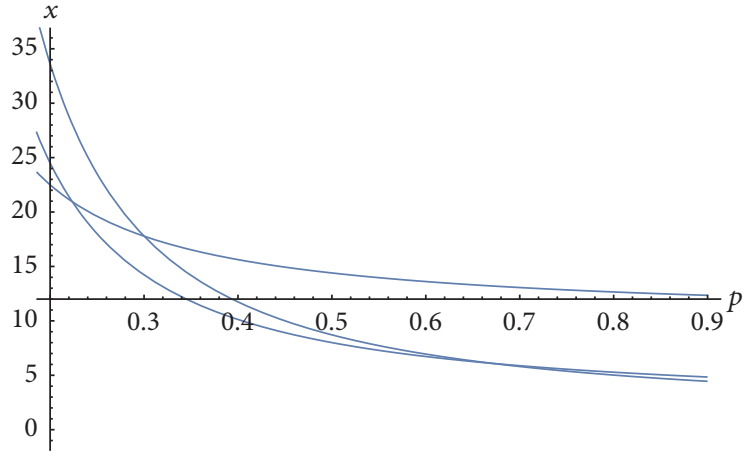

FIGURE 6: Graphs of $x^{*}=x^{*}(p)$ at bifurcation threshold in case of $\mu_{2}=0.1,0.5$, and 0.9. $\mu_{1}=1$. When $\mu_{2}=0.1, x^{*}(0.2) \approx 33, \mu_{2}=0.5$ and 0.9 imply $x^{*}(0.2) \approx 25$ and $x^{*}(0.2) \approx 22$.

which is analysed in [10]. Note that $x_{t}=x_{1 . t}+x_{2, t}$. Thus, the difference between our model and (25) is that while we consider cases where only the mature part of population contributes to density effects, the whole population contributes in (25). This has a pronounced impact on the results. Indeed, just as (2), the fixed point $\left(\widehat{x}_{1}, \widehat{x}_{2}\right)$ of (25) is stable in case of $F$ sufficiently small and loses its hyperbolicity at

$$
\widehat{x}=\frac{2\left(\mu_{1}+\mu_{2}-\mu_{1} p\right)\left(1-\mu_{2}+\mu_{1} p\right)}{\left(1-\mu_{2}\right)\left(1+\mu_{2}-\mu_{1} p\right)\left(1-\mu_{1}(1-p)\right)},
$$

where $\widehat{x}=\widehat{x}_{1}+\widehat{x}_{2}$, but in contrast to our model, $\left(\widehat{x}_{1}, \widehat{x}_{2}\right)$ becomes unstable as the dominant eigenvalue of the linearization of (25) crosses the boundary of the unit circle through -1 . Consequently, when $\hat{x}$ exceeds RHS of (26), a (stable) two-period orbit is established and depending on life history, further increase of $F$ may generate periodic orbits of period $2^{k}, k>1$, as well as chaotic dynamics. Also, note that while all stability curves in Figure 6 are decreasing (independent of $\left.\mu_{2}\right), \widehat{x}(p)$ is an increasing function whenever $\mu_{2}$ is large (cf. [10]). Therefore, based upon the analysis of (25), Neubert and Caswell [10] conjectured that species with precocious iteroparous life histories possess more stable dynamics than 
species with delayed iteroparous life histories. Due to the findings from (2), we feel that this conjecture should be modified.

Let us now focus on possible periodic dynamics. Since $\left(x_{1}^{*}, x_{2}^{*}\right)$ fails to be hyperbolic as a pair of complex valued eigenvalues cross the unit circle, we may exclude periodic dynamics of period $2^{k}, k \geq 1$. Moreover, as is shown, when $F$ exceeds $F_{c}$ and $\left|F-F_{c}\right|$ is small (cf. (9b)), the dynamics is restricted to an invariant curve. On that curve, (2) is topologically equivalent to a circle map which in polar coordinates may be expressed as

$$
\begin{gathered}
(r, \theta) \longrightarrow\left((1+d \mu) r+a r^{3}, \theta+c+b r^{2}\right) \\
+ \text { higher order term }
\end{gathered}
$$

(see [26]), where $c=|\arg \lambda|$ gives asymptotic information of the rotation number associated with the circle map. Now, at threshold (9b), the solution of (6) may be expressed as

$$
\lambda=\frac{\mu_{1}(1-p)+\mu_{2}}{2} \pm i \sqrt{1-\frac{\left(\mu_{1}(1-p)+\mu_{2}\right)^{2}}{4}}
$$

and here we may notice that if $p \rightarrow 1$ and $\mu_{2}$ is small (precocious semelparity), we roughly have $\arg \lambda=\pi / 2$ which in turn implies that the rotation number is close to $1 / 4$. This signals approximate 4-periodic behaviour and one may even expect exact 4-period orbits through frequency locking as $F$ is further increased. Examples of such behaviour may be obtained in [5] or [27]. However, this does not occur. We have not been able to detect periodic dynamics of low period (period 3 or 4) generated by (2). Therefore, based upon our findings here, lots of simulations, and indeed also findings from other stage- and age-structured population models, it appears that if $x_{2}$ only contributes to density effects, periodic dynamics are likely to be absent.

Next, let us turn to predation. The predator population must reach a certain size in order to establish a fixed point $\left(x_{1}^{*}, x_{2}^{*}, y^{*}\right)$ where both species coexist. Depending on parameter values, $\left(x_{1}^{*}, x_{2}^{*}, y^{*}\right)$ may be stable or unstable. Moreover, starting at a low value, an increase of $a y$ acts in a stabilizing fashion, but when ay is large, further enlargement acts in a destabilizing fashion. The "turning" point is where graphs of (15a) and (15d) intersect. This scenario takes place independent of prey life histories.

However, the "strength" of increasing ay is strongly related to life history of prey. Regarding precocious semelparous life history, we have shown that a prey population which possesses nonstationary dynamics may first be stabilized (as shown in Figure 4) and then driven to chaos through an enlargement of predation pressure. In fact (through simulations), we have also verified that a prey population which in the absence of predators exhibits chaotic oscillations may be stabilized and then brought to another chaotic regime as predation pressure is increased. Thus, it is plausible to consider predation pressure as a strong (both stabilizing and destabilizing) effect. It should also be mentioned that the results above are in excellent agreement with the findings obtained from age-structured prey-predator models with few age classes (cf. [21]). In many ways, this is expected. Indeed, if $\mu_{2}$ becomes small and $p \rightarrow 1$, one may argue that stagestructured models degenerate to age-structured models. One important difference between the outcomes of discrete ageand stage-structured models in the precocious semelparous case is linked to possible periodic dynamics. In the agestructured case, it is possible to show that prey that possesses periodic dynamics of low period may force the predator to oscillate with the same kind of periodicity. Such phenomena have not been detected in our model.

The impact of predation when the prey possesses a delayed, semelparous or iteroparous, life history is somewhat different in the sense that the destabilizing effect does not seem to be very strong. It is not obvious what causes this discrepancy. In the precocious case, the value of $y^{*}$ gets smaller but not very much smaller, compared to the delayed case when a is increased. Hence, when $a$ is large, the prey is exposed to a large predator population with excellent hunting skills. This is not the situation in the delayed cases. Although an increase of $a$ leads to an increase of $a y^{*}$ here too, there is a substantial reduction of predators possessing good hunting skills.

\section{Appendix}

\section{A. Proof of Theorem 1 in the Main Text}

As already shown, if $R_{0}>1$, then $\left(\tilde{x}_{1}, \tilde{x}_{2}\right)$ is unstable. Moreover, the restrictions on parameters given in (2) ensure that $A_{0}$ is irreducible and that $A_{x}$ is nonnegative for all $\left(x_{1}, x_{2}\right) \in$ $\mathfrak{R}^{2}$. Consequently, (2) is $\mathfrak{R}^{+} \backslash\{0\}$ forward invariant. We must show that there exists a compact set $X \in \mathfrak{R}^{2}$ such that for all $\left(x_{1,0}, x_{2.0}\right) \in \mathfrak{R}^{2}$ there exists a $t^{M}=t^{M}\left(x_{1,0}, x_{2.0}\right)$ satisfying $x_{t} \in X$ for all $t>t^{M}$. To this end, let $K_{0}$ be a constant satisfying $\exp \left(-x_{2}\right) x_{2} \leq K_{0}$. Then, from (2) (using difference equation notation),

$$
x_{1, t+1} \leq \mu_{1}(1-p) x_{1, t}+F K_{0},
$$

and by induction

$$
x_{1, t+1} \leq\left(\mu_{1}(1-p)\right)^{t} x_{1,0}+F K_{0}\left(1-\mu_{1}(1-p)\right)^{-1} \text {. }
$$

Then, there exists $t^{A}=t^{A}\left(x_{1,0}\right)$ such that for $t>t^{A}$

$$
x_{1, t+1} \leq 2 F K_{0}\left(1-\mu_{1}(1-p)\right)^{-1}=K_{1} \text {. }
$$

Further, in case if $t>t^{A}$, we also have

$$
x_{2, t^{M}} \leq \mu_{1} p K_{1}+\mu_{2} x_{2, t},
$$

and once again (induction) we find that for $t>t^{B}\left(x_{2,0}\right)$

$$
x_{2, t} \leq 2 \mu_{1} p K_{1}\left(1-\mu_{2}\right)^{-1}=K_{2} \text {. }
$$

Finally, take $t^{M}=\max \left\{t^{A}, t^{B}\right\}$ and $K=\max \left\{K_{1}, K_{2}\right\}$; then, for $t>t^{M}, x_{1, t} \leq K_{1}, x_{2, t} \leq K_{2}$, and we are done. This proof is based upon Kon et al. (2004). 


\section{B. Proof of Theorem 2 in the Main Text}

The proof consists of two parts. First we show that the eigenvalues really leave the unit circle at threshold. To this end, note that (6) implies that

$|\lambda|$

$$
=\sqrt{\mu_{1}(1-p) \mu_{2}-(1-K)\left(1-\mu_{2}\right)\left(1-\mu_{1}(1-p)\right)},
$$

hence evaluated at threshold (9b)

$$
\begin{aligned}
\frac{d}{d F}|\lambda| & =\frac{d|\lambda|}{d K} \frac{d K}{d F} \\
& =\frac{1}{2} \mu_{1} p \exp \left(-\frac{2-\left(\mu_{1}(1-p)+\mu_{2}\right)}{\left(1-\mu_{2}\right)\left(1-\mu_{1}(1-p)\right)}\right) \\
& >0 .
\end{aligned}
$$

Thus, the eigenvalues leave the unit circle.

Next, note that the Jacobian of (2) (evaluated at threshold) may be written as

$$
J=\left(\begin{array}{cc}
\mu_{1}(1-p) & \frac{\mu_{1}(1-p)-\mu_{2}-1}{\mu_{1} p} \\
\mu_{1} p & \mu_{2}
\end{array}\right)
$$

with associated complex valued modulus 1 eigenvalues

$$
\lambda=\frac{\mu_{1}(1-p)+\mu_{2}}{2} \pm \frac{b}{2} i
$$

where $b=\sqrt{4-\left(\mu_{1}(1-p)+\mu_{2}\right)^{2}}$.

Define the matrix

$$
T=\left(\begin{array}{cc}
\frac{\mu_{1}(1-p)-\mu_{2}}{2 \mu_{1} p} & -\frac{b}{2 \mu_{1} p} \\
1 & 0
\end{array}\right)
$$

whose columns are the real and imaginary parts, respectively, of the eigenvectors associated with the eigenvalues.

Then, after expanding the first component of (2) up to the third order, applying the change of coordinates $\left(\widehat{x}_{1}, \widehat{x}_{2}\right)=$ $\left(x_{1}-x_{1}^{*}, x_{2}-x_{2}^{*}\right)$ (in order to transform the bifurcation to the origin) together with the transformations

$$
\left(\begin{array}{l}
\hat{x}_{1} \\
\hat{x}_{2}
\end{array}\right)=T\left(\begin{array}{l}
u \\
v
\end{array}\right)\left(\begin{array}{l}
u \\
v
\end{array}\right)=T^{-1}\left(\begin{array}{l}
\hat{x}_{1} \\
\hat{x}_{2}
\end{array}\right),
$$

map (4) may be cast into "standard form" as

$$
\begin{aligned}
\left(\begin{array}{l}
u \\
v
\end{array}\right) & \\
\longrightarrow & \left(\begin{array}{cc}
\frac{\mu_{1}(1-p)+\mu_{2}}{2} & -\frac{b}{2} \\
\frac{b}{2} & \frac{\mu_{1}(1-p)+\mu_{2}}{2}
\end{array}\right)\left(\begin{array}{l}
u \\
v
\end{array}\right) \\
& +\left(\begin{array}{c}
0 \\
g(u)
\end{array}\right),
\end{aligned}
$$

where

$$
\begin{aligned}
g(u) & =-\frac{2 \mu_{1} p}{b}\left(A u^{2}+B u^{3}\right), \\
A & =f^{\prime}+\frac{1}{2} f^{\prime \prime} x_{2}^{*} \\
& =\frac{1}{2 \mu_{1} p}\left\{\mu_{1}(1+p)+\mu_{2}-2 \mu_{1}(1-p) \mu_{2}\right\} \\
& =\frac{1}{2 \mu_{1} p} \widetilde{A}, \\
B & =\frac{1}{2} f^{\prime \prime}+\frac{1}{6} f^{\prime \prime \prime} x_{2}^{*} \\
& =\frac{1}{6 \mu_{1} p}\left\{1-2 \mu_{1}(1-p)-2 \mu_{2}+3 \mu_{1}(1-p) \mu_{2}\right\} \\
& =\frac{1}{6 \mu_{1} p} \widetilde{B},
\end{aligned}
$$

and $f=F \exp \left(-x_{2}\right)$.

Now, by use of Theorem 3.5.2 in Guckenheimer and Holmes (1990), the bifurcation will be of supercritical nature if the quantity a defined through

$$
\begin{aligned}
a= & -\operatorname{Re}\left[\frac{(1-2 \lambda) \bar{\lambda}^{2}}{1-\lambda} \xi_{11} \xi_{20}\right]-\frac{1}{2}\left|\xi_{11}\right|^{2}-\left|\xi_{02}\right|^{2} \\
& +\operatorname{Re}\left(\bar{\lambda} \xi_{21}\right)
\end{aligned}
$$

is negative. For the problem at hand,

$$
\begin{aligned}
\xi_{20} & =\frac{1}{8} i g_{u u}=-\frac{\mu_{1} p}{2 b} A i \\
\xi_{11} & =\frac{1}{4} i g_{u u}=-\frac{\mu_{1} p}{b} A i \\
\xi_{02} & =\frac{1}{8} i g_{u u}=-\frac{\mu_{1} p}{2 b} A i \\
\xi_{21} & =\frac{1}{16} i g_{u u u}=-\frac{3 \mu_{1} p}{4 b} B i \\
\operatorname{Re}[ & {\left[\frac{(1-2 \lambda) \bar{\lambda}^{2}}{1-\lambda} \xi_{11} \xi_{20}\right] } \\
& =\frac{1}{4}\left(\frac{\mu_{1} p}{b} A\right)^{2}\left(3+\left(\mu_{1}(1-p)+\mu_{2}\right)\right) .
\end{aligned}
$$

This yields

$$
a=-\frac{1}{16}\left\{\left(\frac{\widetilde{A}}{b}\right)^{2}\left(\mu_{1}(1-p)+\mu_{2}+6\right)+\widetilde{B}\right\},
$$

which is negative and we conclude that the bifurcation is supercritical.

\section{Conflicts of Interest}

The authors declare that they have no conflicts of interest. 


\section{Acknowledgments}

The publication charges for this article have been covered by a grant from the publication fund of UiT The Arctic University of Norway.

\section{References}

[1] J. Guckenheimer, G. Oster, and A. Ipaktchi, "The dynamics of density dependent population models," Journal of Mathematical Biology, vol. 4, no. 2, pp. 101-147, 1977.

[2] S. A. Levin and C. P. Goodyear, "Analysis of an age-structured fishery model," Journal of Mathematical Biology, vol. 9, no. 3, pp. 245-274, 1980.

[3] J. A. Silva and T. G. Hallam, "Compensation and stability in nonlinear matrix models," Mathematical Biosciences, vol. 110, no. 1, pp. 67-101, 1992.

[4] J. A. Silva and T. G. Hallam, "Effects of delay, truncations and density dependence in reproduction schedules on stability of nonlinear Leslie matrix models," Journal of Mathematical Biology, vol. 31, no. 4, pp. 367-395, 1993.

[5] A. Wikan and E. Mjølhus, "Periodicity of 4 in age-structured population models with density dependence," Journal of Theoretical Biology, vol. 173, no. 2, pp. 109-119, 1995.

[6] A. Wikan and E. Mjølhus, "Overcompensatory recruitment and generation delay in discrete age-structured population models," Journal of Mathematical Biology, vol. 35, no. 2, pp. 195-239, 1996.

[7] E. Mjølhus, A. Wikan, and T. Solberg, "On synchronization in semelparous populations," Journal of Mathematical Biology, vol. 50, no. 1, pp. 1-21, 2005.

[8] J. M. Cushing, B. Dennis, R. A. Desharnais, and R. F. Costantino, "An interdisciplinary approach to understanding nonlinear ecological dynamics," Ecological Modelling, vol. 92, no. 2-3, pp. 111-119, 1996.

[9] R. F. Costantino, R. A. Desharnais, J. M. Cushing, and B. Dennis, "Chaotic dynamics in an insect population," Science, vol. 275, no. 5298, pp. 389-391, 1997.

[10] M. G. Neubert and H. Caswell, "Density-dependent vital rates and their population dynamic consequences," Journal of Mathematical Biology, vol. 41, no. 2, pp. 103-121, 2000.

[11] N. V. Davydova, O. Diekmann, and S. A. van Gils, "Year class coexistence or competitive exclusion for strict biennials?" Journal of Mathematical Biology, vol. 46, no. 2, pp. 95-131, 2003.

[12] A. Wikan and A. Eide, "An analysis of a nonlinear stagestructured cannibalism model with application to the northeast arctic cod stock," Bulletin of Mathematical Biology, vol. 66, no. 6, pp. 1685-1704, 2004.

[13] A. Wikan, "On the interplay between cannibalism and harvest in stage-structured population models," Journal of Marine Biology, vol. 2015, Article ID 580520, 8 pages, 2015.

[14] A. Wikan, "Age or stage structure? A comparison of dynamic outcomes from discrete age- and stage-structured population models," Bulletin of Mathematical Biology, vol. 74, no. 6, pp. 1354-1378, 2012.

[15] M. Gyllenberg, G. Söderbacka, and S. Ericsson, "Does migration stabilize local population dynamics? Analysis of a discrete metapopulation model," Mathematical Biosciences, vol. 118, no. 1, pp. 25-49, 1993.

[16] A. Wikan, "On reserves, stability and the maximum sustainable yield problem," Journal of Mathematics and Statistics, vol. 9, no. 4, pp. 325-333, 2013.
[17] J. R. Beddington, C. A. Free, and J. H. Lawton, "Dynamic complexity in predator-prey models framed in difference equations," Nature, vol. 255, no. 5503, pp. 58-60, 1975.

[18] A. Hastings, "Age-dependent predation is not a simple process. II. Wolves, ungulates, and a discrete time model for predation on juveniles with a stabilizing tail," Theoretical Population Biology, vol. 26, no. 2, pp. 271-282, 1984.

[19] H. A. Lauwerier and J. A. Metz, "Hopf bifurcation in hostparasitoid models," IMA Journal of Mathematics Applied in Medicine and Biology, vol. 3, no. 3, pp. 191-209, 1986.

[20] M. G. Neubert and M. Kot, "The subcritical collapse of predator populations in discrete-time predator-prey models," Mathematical Biosciences, vol. 110, no. 1, pp. 45-66, 1992.

[21] A. Wikan, "From chaos to chaos. An analysis of a discrete age-structured prey-predator model," Journal of Mathematical Biology, vol. 43, no. 6, pp. 471-500, 2001.

[22] S. A. Gourley and Y. Kuang, "A stage structured predator-prey model and its dependence on maturation delay and death rate," Journal of Mathematical Biology, vol. 49, no. 2, pp. 188-200, 2004.

[23] H. Behncke, "Periodical cicadas," Journal of Mathematical Biology, vol. 40, no. 5, pp. 413-431, 2000.

[24] R. Kon, Y. Saito, and Y. Takeuchi, "Permanence of single-species stage-structured models," Journal of Mathematical Biology, vol. 48, no. 5, pp. 515-528, 2004.

[25] J. D. Murray, Mathematical Biology: Spatial Models and Biomedical Applications, Biomathematics, Springer, Berlin, Germany, 3rd edition, 1993.

[26] J. Guckenheimer and P. Holmes, Nonlinear Oscillations, Dynamical Systems, and Bifurcations of Vector Fields, Springer, New York, NY, USA, 1990.

[27] A. Wikan, "Dynamic consequences of reproductive delay in Leslie matrix models with nonlinear survival probabilities," Mathematical Biosciences, vol. 146, no. 1, pp. 37-62, 1997. 


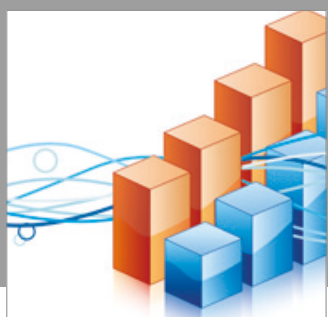

Advances in

Operations Research

vatersals

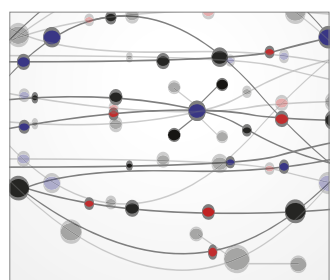

\section{The Scientific} World Journal
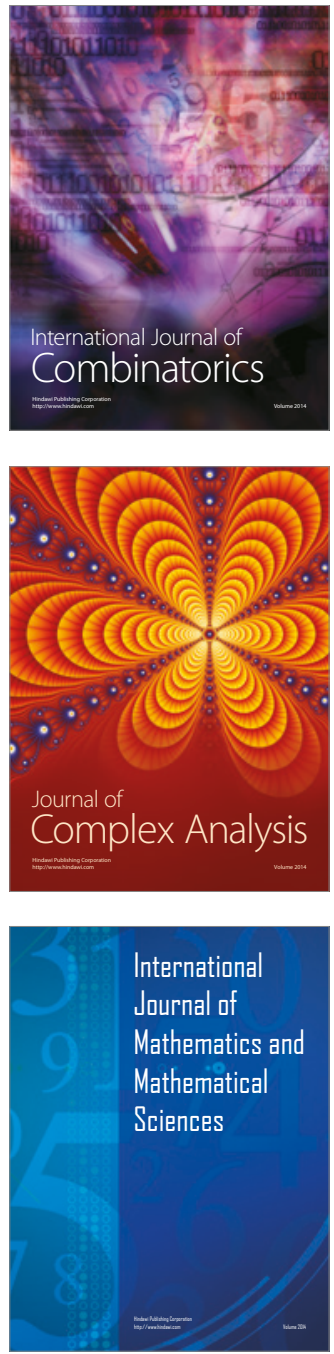
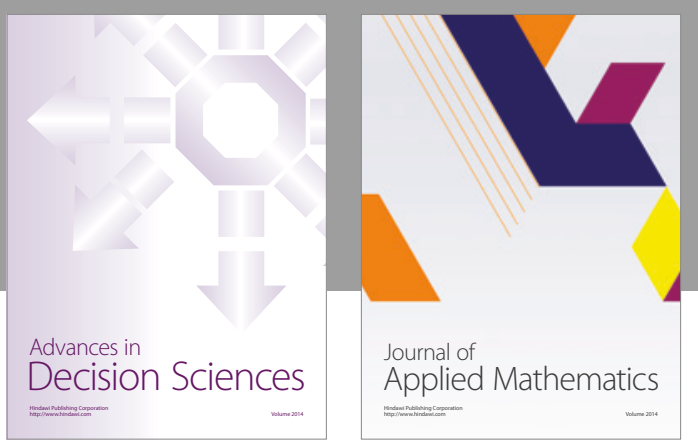

Algebra

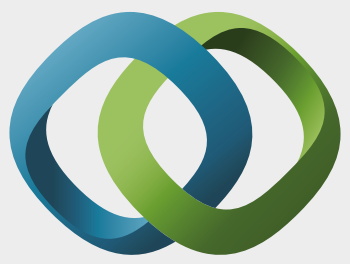

\section{Hindawi}

Submit your manuscripts at

https://www.hindawi.com
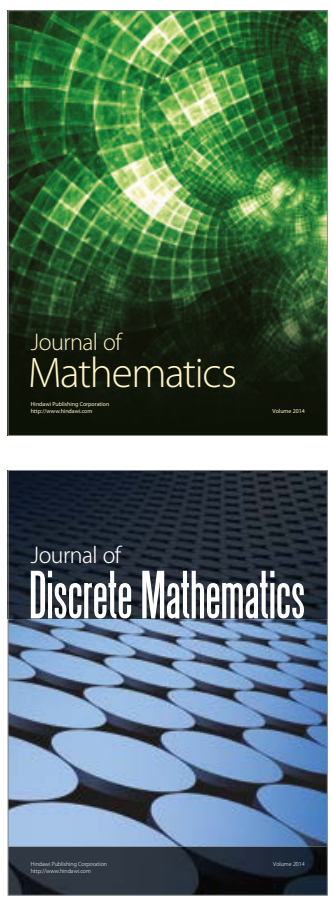

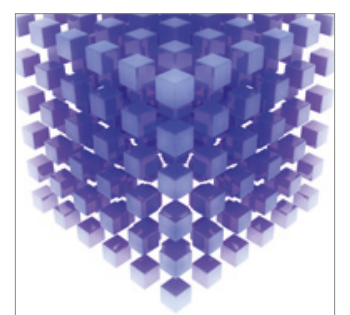

Mathematical Problems in Engineering
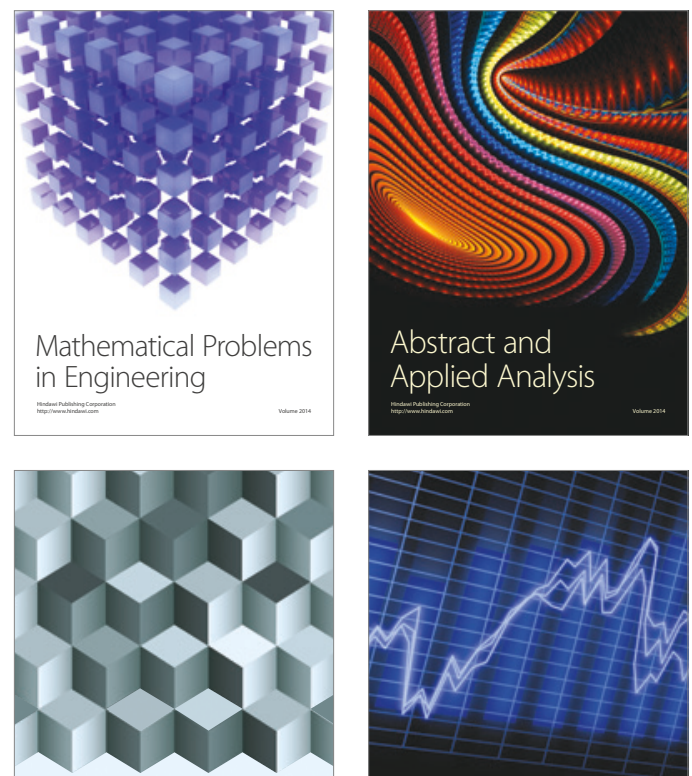

Journal of

Function Spaces

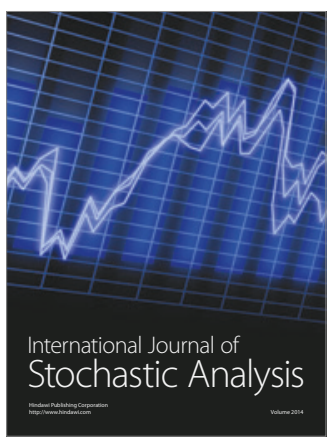

Probability and Statistics
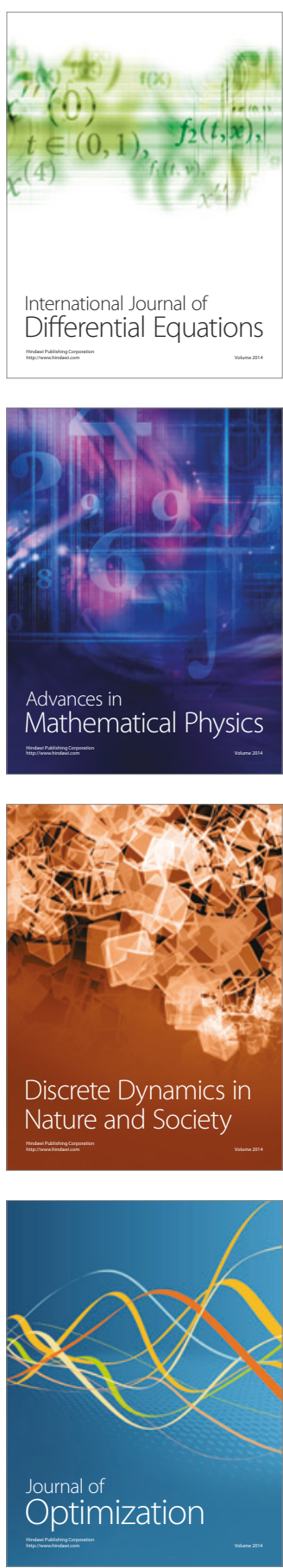